\title{
SpaCetime Physics AND Advanced Propulsion Concepts
}

AIAA 2006-4608

\author{
Walter Dröscher ${ }^{1}$, Jochem Hauser ${ }^{1,2}$
}

\author{
${ }^{1}$ Institut für Grenzgebiete der Wissenschaft, Innsbruck, Austria \\ and
}

\section{${ }^{2}$ Faculty Karl-Scharfenberg, University of Applied Sciences, Salzgitter, Germany \\ Short Version}

Abstract: Spacetime physics includes general relativity $(G R)$, quantum theory, quantum gravity, string theory (additional external dimensions), and gauge theory (additional internal dimensions) as well as some modern variations. The paper will discuss the requirements on future propulsion systems stemming from the demands for routine missions to $L E O$, the moon, or planetary missions within the solar system, as well as interstellar flight. These requirements are compared with the limits imposed by the physical laws of $G R$ in conjunction with the physical theories listed above. The physical consequences of these field theories in curved-spacetime as well as string and gauge theory, are discussed. Moreover, recent developments in the structure of spacetime are presented, and their consequences for advanced propulsion systems are outlined. In particular, a novel experiment (ESA, March 2006) reporting about the generation of an artificial gravitational field in the laboratory is discussed. This experiment, if confirmed, could serve as the basis for a field propulsion device. Since a thorough understanding of the underlying physical principle is of prevailing importance, a detailed theoretical analysis of this experiment is presented. Utilizing the experimental data along with the insight gained from theoretical considerations, a concept for a field propulsion device is developed. Preliminary results on the capability of this device will be given. Finally, an outlook of the necessary experimental and theoretical prerequisites is given to both understand the novel physics as well as the technical requirements for such a propulsion device.

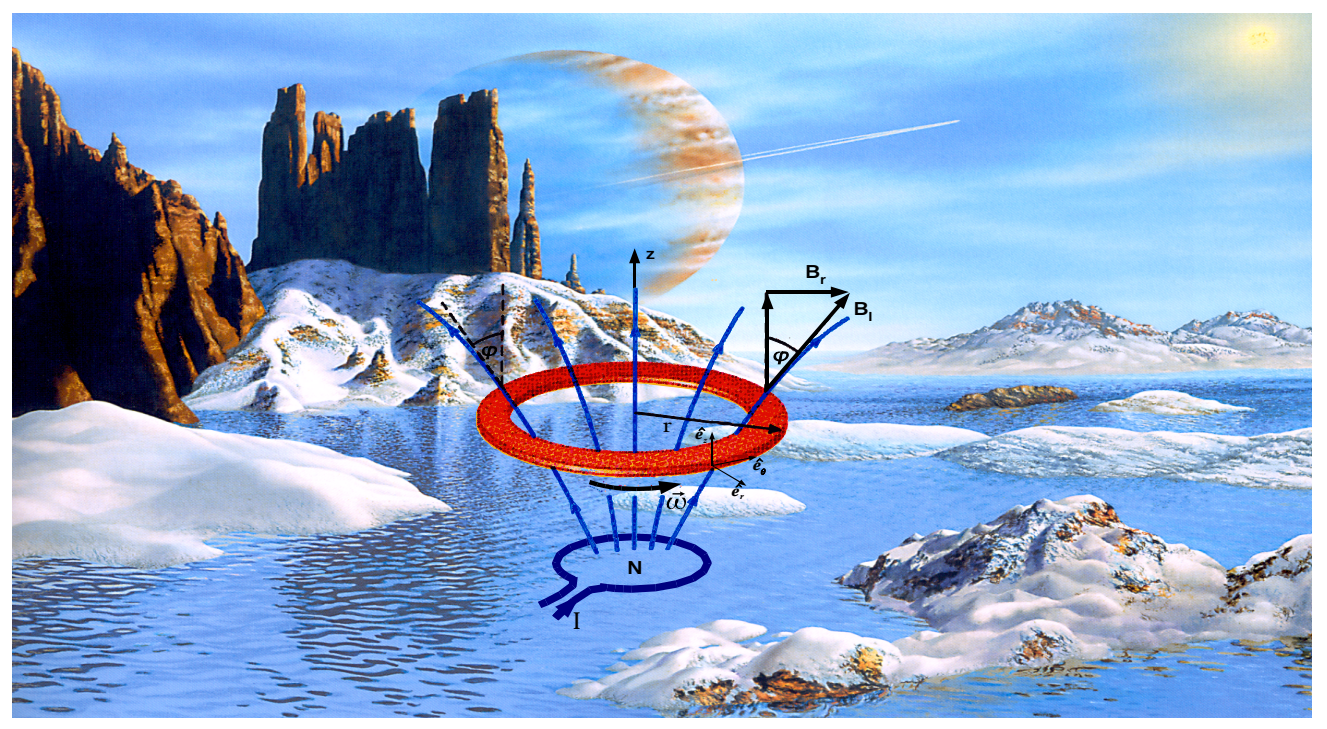

Senior scientist, ${ }^{2}$ Senior member AIAA, member SSE, (C) IGW, Innsbruck, Austria 2006 


\section{Spacetime and Space Propulsion ${ }^{2}$}

Space flight within the solar system requires the covering of large distances. The distance to our moon is some $3.8 \times 10^{5} \mathrm{~km}$, while Mars, our favorite destination is some 0.5 A.U. away (astronomical units, 1 A.U. $=1.5 \times 10^{8} \mathrm{~km}$ ). The next planet, Jupiter, is already some 4 A.U. away from Earth. The closest star is Proxima centauri, which is $1.30 \mathrm{pc}$ away from earth (parsec, $1 \mathrm{pc}=3.3 \mathrm{ly}$ ) or, using lightyear, the distance light travels in the time of 1 year, $\left(1 \mathrm{ly}=9.46 \times 10^{12} \mathrm{~km}\right)$, it would take the light some 4.3 years to reach this star. Expressed in miles, the distance is some 25 trillion miles from earth. The star closest to us which is similar to our sun with respect to size and surface temperature is Centauri, some 1.33 pc away. But these distances are small when compared to the dimension of the Milky Way Galaxy which comprises a galactic disk of some 100,000 ly in diameter and some 4,000 ly for the galactic bulge. Our solar system is located some 8 kpc (kilo parsec) from the galactic center. Our galaxy contains some 100 billion stars, and the universe contains some 100 billion galaxies. The farthest of these galaxies is some 13 billion ly away, which is roughly the size of the observable universe. The age of the Earth is estimated to be some 4.5 billion years, while there are stars that are 7 to 10 billion years old. Having mentioned both distance and time, the concept of spacetime has been utilized and also, implicitly, the concept of metric has been employed to measure distances in this four-dimensional spacetime. This is the environment in which spaceflight has to take place.

Next, we will briefly discuss our current capabilities ${ }^{3}$ to travel through space and time that is, in spacetime. Current space transportation systems are based on the principle of momentum generation, regardless whether they are chemical, electric, plasma-dynamic, nuclear (fission) or fusion, antimatter, photonic propulsion (relativistic) and photon driven (solar) sails, or exotic Bussard fusion ramjets, solar sails, nuclear explosions (pusher, Orion), antimatter propulsion are most likely in the realm of unfeasible technologies because of the large engineering and/or safety problems as well as their prohibitively high cost. The specific impulse achievable from thermal systems ranges from some $500 \mathrm{~s}$ for advanced chemical propellants (excluding free radicals or metastable atoms), some

2 Invited paper in the session 50-NFF-3 Faster Than Light, AIAA $42^{\text {nd }}$ Joint Propulsion Conference, Sacramento, CA, 9-12 July 2006. A more detailed paper will be available at the conference.

3 The cover picture shows a combination of two pictures. The first one, taken from ref. [1], shows a view (artist's impression) from an existing planet orbiting the solar-type star HD 222582 some 137 ly away. The second one depicts the principle of the propulsion system used to reach this planet, see Fig. 3.
$1,000 \mathrm{~s}$ for a fission solid-core rocket (NERVA program [2]) using hydrogen as propellant (for a gas-core nuclear rocket specific impulse could be 3,000 s or higher but requiring very high pressures) up to $200,000 \mathrm{~s}$ for a fusion rocket [3]. Although recently progress was reported in the design of nuclear reactors for plasma propulsion systems [4] such a multimegawatt reactor has a mass of some $3 \times 10^{6} \mathrm{~kg}$ and, despite high specific impulse, has a low thrust to mass ratio, and thus is most likely not capable of lifting a vehicle from the surface of the earth. With regard to fusion propulsion, the gasdynamic mirror has been proposed as highly efficient fusion rocket engine. However, recent experiments revealed magneto-hydrodynamic instabilities [5] that make such a system questionable even from a physics standpoint, since magnetohydrodynamic stability has been the key issue in fusion for decades. The momentum principle combined with the usage of fuel, because of its inherent physical limitations, will not allow going to routine spaceflight. The above discussion does not even consider the difficulties entered when the simplicity of the physical concept meets the complexities of the workable propulsion system.

At relativistic speeds, Lorentz transformation replaces Galilei transformation that is, the rest mass of the propellant is multiplied by the factor $\left(1-v^{2} / c^{2}\right)$ that goes to infinity if the exhaust velocity $v$ equals $c$, the speed of light in vacuum.

For instance, a flight to the nearest star at a velocity of some $16 \mathrm{~km} / \mathrm{s}$ would take about 80,000 years. If the speed of light cannot be transcended, interstellar travel is impossible. We conclude with a phrase from the recent book on future propulsion by Czysz and Bruno [6] : If that remains the case, we are trapped within the environs of the Solar System. In addition, the current state of propulsion does not allow either convenient interplanetary travel and inflicts prohibitively high cost even for low earth orbits.

As mentioned by Krauss [7], general relativity $(G R)$ allows metric engineering, including the so-called warp drive, see Sec. 2.2, but superluminal travel would require negative energy densities. Furthermore, in order to tell space to contract (warp), a signal is necessary that, in turn, can travel only with the speed of light. $G R$ therefore does not allow this kind of travel.

On the other hand, current physics is far from providing final answers. First, there is no unified theory that combines $G R$ and $Q M$ (quantum mechanics). Second, not even the question about the total number of fundamental interactions can be answered. Hence, the goal to find a unified field theory is a viable undertaking, because it 
might lead to novel physics, which, in turn, might allow for a totally different principle in space transportation. ${ }^{4}$

\section{Classical Spacetime}

In $G R$ the model of space and time supports continuous and differentiable functions and provides a structure that has the same local topology as $\mathbb{R}^{4}$. Therefore, spacetime is a topological space and thus comprises a collection of open sets. For small regions it is assumed that the open sets possess the topology of $\mathbb{R}^{4}$. Therefore, a one-to-one mapping exists between the open set of spacetime and $\mathbb{R}^{4}$. Each point in spacetime has a unique image in $\mathbb{R}^{4}$ and vice versa.

\subsection{Spacetime as a Manifold}

Equipped with the features described above, spacetime is called a manifold. In general, physical fields defined on an open set of this manifold are assumed to be differentiable. Spacetime thus is considered to be a multiply differentiable manifold. However, as will be shown in Sec. 3, spacetime must be quantized. Therefore, it is not generally possible to have a third point between any two points in spacetime. Spacetime is not dense and hence the concept of manifold is incorrect, at least on the Planck length, see below. In $S R T$ (special theory of relativity) Lorentz contraction is continuous, but this contradicts the concept of minimum length.

At Planck scales $S R T$ cannot be correct. GR uses the concept of curvature, but at Planck scales it cannot be measured exactly. This is equivalent to fluctuations of curvature and thus of gravitation itself. A unified field theory describing all physical interactions by individual metrics would be subject to fluctuations as well that is, all physical forces would be subject to these fluctuations.

Physics is not possible below the Planck scale, since concepts of metric, dimensionality, or points are not defined. Spacetime itself is a field and thus needs to be quantized, leading to quantum gravity $(Q G)$, see, for instance [8]. So far, $Q G$ has not lead to a unified field theory, and does not predict phenomena that could lead to a novel propulsion concept. Conventional wisdom claims that quantized spacetime acts on the Planck scale only. On macroscopic scales the concepts of $G R$ are sufficient to describe spacetime. However, this argument may turn out to be invalid, since despite the smallness of the quantized action, denoted by the Planck constant $\hbar$, physical phenomena on the macroscopic scale do occur, for instance superconducting. Therefore, it is possible that a quantized spacetime will lead to observable physical phenomena. A quantized spacetime together with the prediction of a repulsive gravitational force, predicted by the unified theory presented, Sec. 3.2, leads to the concept of hyperspace (or parallel space) in which the limiting speed of light is $n c$, with $n>$ 1 , integer and $c$ the vacuum speed of light [9], [10].

4 A more detailed discussion will be given in our paper entitled Field Propulsion I: Novel Concepts for Space Propulsion.

\subsection{The Physics of Continuous Spacetime}

Einsteinian spacetime [11], [12] is indefinitely divisible and can be described by a differentiable manifold. In reality, however, spacetime is a quantized field. Gra-vitation is the dominant force in systems on astronomical scales. $G R$ can be summarized in the single sentence: matter curves spacetime. For a flat geometry, the angles of a triangle add up to 180 degrees. The spacetime metric of a flat universe is given by

$$
d s^{2}=d x^{2}+d y^{2}+d z^{2}-c^{2} d t^{2} .
$$

On the surface of a sphere spherical coordinates are used

$$
d s^{2}=d r^{2}+r^{2} d \theta^{2}+r^{2} \sin ^{2} \theta d \phi^{2}-c^{2} d t^{2} .
$$

For a generally curved spacetime the metric is written in the form (double indices are summed over)

$$
d s^{2}=g_{\mu \nu} d x^{\mu} d x^{v}
$$

where $g_{\mu \nu}$ is the metric, $x^{1}, x^{2}, x^{3}$ are the spatial coordinates, and $x^{4}$ is the time coordinate ${ }^{5}$. The cosmo-logical principle states that the Universe does not have preferred locations (homogeneous) or directions (isotropic). Therefore the spatial part of the metric has constant curvature. Extending the spherical metric, the most general metric is given by the Robertson-Walker metric

$$
d s^{2}=a^{2}(t)\left[\frac{d r^{2}}{1-k r^{2}}+r^{2}\left(d \theta^{2}+\sin ^{2} \theta d \phi^{2}\right)\right]-c^{2} d t^{2},
$$

where $a(t)$ is the scale factor of the Universe. Here it is assumed that the Universe started from a fixed size $x_{0}$ and expanded according to $a(t)$. Two points that were at distance $x_{0}$ at time $t_{0}$, now are at distance $x(t)=a(t) x_{0}$.

In 1994 Alcubierre [13], [14] specified the following metric, termed the warp-drive spacetime

$$
d s^{2}=\left[d x^{2}-V_{s} f\left(r_{s}\right) d t^{2}+d y^{2}+d z^{2}\right]-c^{2} d t^{2},
$$

where $V_{s}(t)$ is the velocity along a given curve $x_{s}(t)^{6}$ and $r_{s}^{2}(t)=\left(x-x_{s}(t)\right)^{2}+y^{2}+z^{2}$. A choice for $f_{s}(t)$ is $f_{s}=\left(1-r_{s} / R\right)^{4}$ and $R$ is a distance. Without proof it is stated that, if this warp-drive metric could be generated - the term metric engineering was coined - around a spaceship, the vehicle would be traveling faster than the speed of light, seen from a spacetime diagram of flat space. Locally the ship is moving less than the speed of light. A bubble of spacetime curvature must surround the spaceship. Since the Alcubierre metric requires a negative local energy density, it cannot work in $G R$. Quantum mechanics allows negative energy density, and perhaps a combination with the quintessence particle, see Fig. 2, the sixth fundamental force predicted by $E H T$. It is interesting to note that the experi-

5 Often the time coordinate is denoted as $x^{0}$.

6 For simplicity $y=0$ and $z=0$ are assumed. 
ment by Tajmar et al. [15] could be interpreted as metric engineering since an artificial gravitational field was generated and, as a result, the local metric has been changed.

There are also spacetime concepts of higher dimen-sionality. Kaluza (1921) introduced an additional fourth spatial dimension into Einstein's field equations, and in a letter to Einstein pointed out that Maxwell's theory of electromagnetism was comprised in the now 5-dimen-sional Einstein equations. However, his theory produced divergencies and could not answer the question about the visibility of this $5^{\text {th }}$ dimension. In 1926 Klein, a Swedish physicist, introduced the concept of a curled up dimension that exists on the Planck length scale only, and thus cannot be observed by experiment. String theory [16], see Sec. 3.1, has extended this concept by intro-ducing 8 additional spatial dimensions, resulting in a total of 11 spatial dimensions.

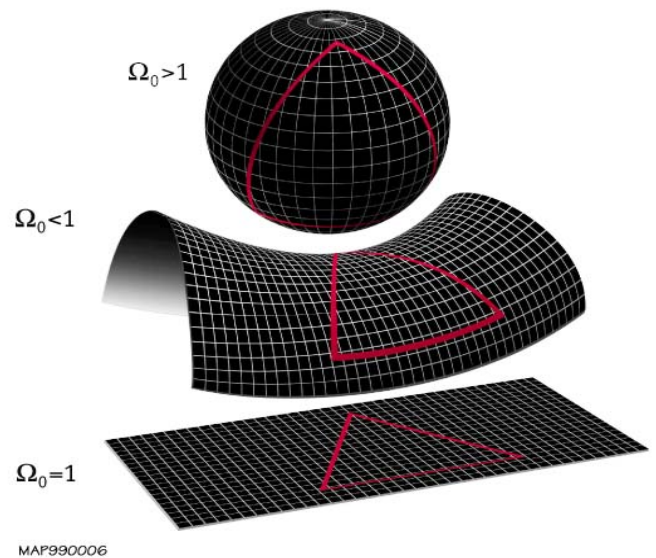

Figure 1: This picture, taken from Wikipedia, shows three types of possible geometries for the Universe, namely closed, open, or flat. At present, a flat Universe is assumed.

\section{Quantized Spacetime}

In the following it is shown that the combination of quantum theory (Heisenberg's uncertainty relation) with special relativity (constancy of the speed of light and $E=m c^{2}$ ) and general relativity (Schwarzschild radius) directly leads to a quantized spacetime, resulting in the well known Planck scales. The proof is straightforward and is given below. The quantization of spacetime leads to the proposition of a hyperspace (parallel space) in which superluminal speeds should be possible, see [17].

Heisenberg's indeterminacy (uncertainty) relation, for instance relating time and energy indeterminacies,

$\Delta t \Delta E>\hbar$, allows for arbitrarily small $\Delta t$ by making the energy uncertainty arbitrarily large. However, this is not the case in the real physical world. It is straight-forward to prove the discreteness of spacetime. To this end, the time measurement process using clocks is analyzed [18]. Einstein's $G R$ itself is used to disprove the existence of continuous spacetime. According to Einstein, the ener- gy of any material object is $E=m c^{2}$. The smallest time interval, $\delta t$, that can be measured must of course be larger than the time uncertainty required to satisfy Heisenberg's uncertainty relation that is $\delta t>\Delta t=\hbar / \Delta E$. A clock of mass $m$ cannot have an energy uncertainty $\Delta E>m c^{2}$, because this would lead to the creation of additional clocks, hence $\delta t>\Delta t=\hbar / m c^{2}$. A clock of length $l$ needs a measuring time $c \delta t>l$ in order to receive the measuring signal. A characteristic length of a material body is its Schwarzschild radius, namely when its gravitational energy equals its total energy $m c^{2}$, i.e., $r_{S}=G m / c^{2}$. This means for the mass of the clock $m<r_{S} c^{2} / G$, because the body must not be a black hole from which signals cannot escape. Inserting the value $l$ for $r_{S}, m<\delta t c^{3} / G$. Inserting the value of $m$ in the above relation for $\delta t$, one obtains the final relation $\delta t^{2}>\hbar G / c^{5}$. Thus the quantization aspect of the GODQ principle, which comprises the four basic physical principles of Nature, see Sec. 3.2.1, directly delivers a fundamental lowest limit for a time interval, termed the Planck time. In a similar way the smallest units for length and mass can be found. As shown above, Planck units are constructed from the three fundamental constants in Nature, namely $\hbar, c$, and $G$. The values for the Planck units are:

$$
\begin{aligned}
& \text { - Planck mass } m_{p}=(c / G)^{1 / 2}=2.176 \times 10^{-8} \mathrm{~kg}, \\
& \text { - } \quad \text { Planck length } l_{p}=\left(G \hbar c^{-3}\right)^{1 / 2}=1.615 \times 10^{-35} \mathrm{~m}, \\
& \text { - } \quad \text { Planck time } t_{p}=\left(G \hbar / c^{5}\right)^{1 / 2}=5.389 \times 10^{-44} \mathrm{~s} .
\end{aligned}
$$

This means that the classical picture of points in a continuous spacetime does not make physical sense. Physics below the Planck units is not possible, since one cannot distinguish between vacuum and matter. No measurements are possible. The nature of spacetime is discrete in the same way as energy is discrete, expressed by $E=h v$. Since spacetime therefore is a quantum field, it should have corresponding quantum states, described by a quantum field theory. Since spacetime is equivalent to gravity, gravity itself needs to be described by a quantum field theory. In both classical physics and quantum mechanics point particles are used, and the inverse force law leads to infinities of type $1 / 0$ at the location of the particle. As was shown above, any particle must have a discrete geometric structure, since it is finite in size. The minimal surface must be proportional to the Planck length squared. From scattering experiments, however, it is known that many particles have a much larger radius, for instance, the proton radius is some $10^{-15} \mathrm{~m}$, and thus its surface would be covered by about $10^{40}$ elemental Planck surfaces. Hence, an elementary particle must be a highly organized and also complex geometrical structure. It is therefore mandatory that point particles are banished. In addition, the organizational state of an entity or structure needs to be measured and therefore, among other reasons, the concept of an organization coordinate in an internal space (de- 
scribing the additional degrees of freedom) is introduced, see Sec. 3.2.1.

\subsection{Spacetime of Higher Spatial Dimensions: String Theory}

The theory by Kaluza and Klein $(1921,1926)$ already introduced a fourth spatial dimension to account for electromagnetism. According to string theory, electrons are not point particles, but are vibrations of a string, whose length is at the Planck scale, some $10^{-35} \mathrm{~m}$. Strings are one-dimensional entities. Modifying the vibrations frequency of these strings they can turn into other particles, for instance, the electron can turn into a neutrino, or into any of the known subatomic particles. String theory leads to a unification of the four fundamental interactions, but requires 11 real spatial dimensions, [16]. However, because of the discrete nature of spacetime there seems to be no need for string theory, which replaces point particles by strings, but requires hitherto unobserved additional spatial dimensions.

However, there is a fundamental difference compared to the concept of spacetime with internal dimensions, in that strings are objects in spacetime, while in the next section a geometrization concept is employed that explains all particles as geometric objects constructed from spacetime itself.

\subsection{Spacetime with Internal Dimensions}

However, there exists another concept, coming from the idea that elementary particles have additional degrees of freedom in some kind of internal space. Therefore, the concept of physical space as the combination of spacetime and internal space is introduced. This marriage of 4dimensional spacetime with internal space is called fiber bundle space mathematically. In the following the term physical space will be used for this combination, since all the fundamental forces of physics will be described in this space. These internal degrees of freedom can then be connected with the dynamical motion in spacetime. This is the geometrical structure utilized in gauge theory. The dimension of the internal space and its symmetries determine the physics that is possible. In order to have a unified field theory the proper internal space has to be constructed that encompasses all interactions of physics. In the next section, $G R$ is equipped with an 8-dimensional internal space (all internal coordinates have negative signature), termed Heim space. Once this internal space is included, all physical interactions are fixed. There is only one single selection rule used to selecting internal subspaces. It turns out that six fundamental physical interactions should exist.

\subsubsection{Extended Heim Theory}

In $E H T$ a set of 8 additional coordinates is introduced, but contrary to String theory, the theory postulates an internal space with 8 dimensions that governs physical events in our spacetime (actually a manifold $\mathrm{M}^{4}$ ). The crucial point lies in the construction of the internal space that should come from basic physical assumptions, which must be generally acceptable. In $E H T$, an 8-dimensional space is constructed, termed Heim space, $\mathrm{H}^{8}$ that is missing in $G R$. In other words, $G R$ does not possess any internal space, and thus has a very limited geometrical structure, namely that of pure spacetime. Because of this limitation, $G R$ cannot describe the fundamental forces in physics and consequently has to be extended. The extension as done in $E H T$, lies in the introduction of the internal space $\mathrm{H}^{8}$. $E H T$ reduces to $G R$ when this internal space is omitted. The metric tensor, as used in $G R$, has purely geometrical means that is of immaterial character only, and does not represent any physics. Consequently, the Einsteinian Geometrization Principle (EGP) is equating the Einstein curvature tensor, constructed from the metric tensor, with the stress tensor, representing energy distribution. Stated in simple terms: matter curves spacetime. In this way, the metric tensor field has become a physical object whose behavior is governed by an action principle, like that of other physical entities.

\section{Six Fundamental Physical Forces}

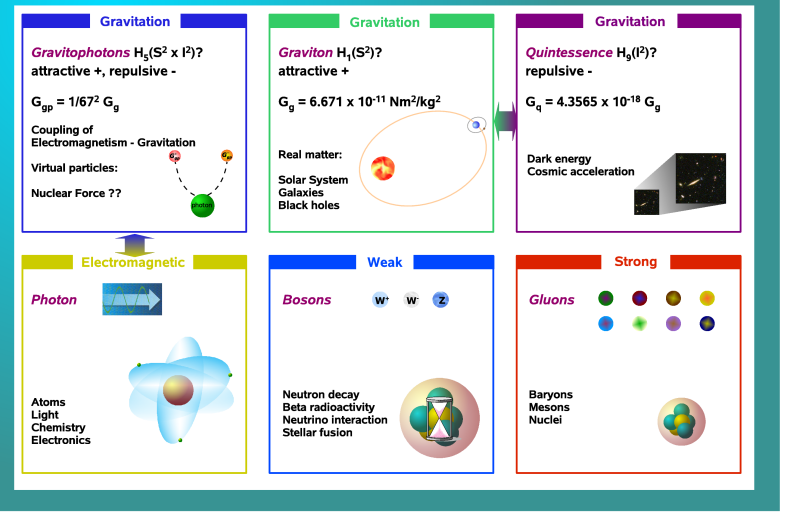

Figure 2: EHT has, as one of its most important consequences, the prediction of two additional, gravitational like interactions and the existence of two messenger particles, termed gravitophoton and quintessence.

According to the quantization principle, the minimal length in the space part of $\mathrm{H}^{8}$ is the Planck length. Applying the geometrization rule of the GODQ principle, see below, Planck mass and Planck time are converted into length units leading to two additional lengths constants $l_{p m}=\hbar / m_{\mathrm{p}} c$ and $l_{p t}=c t_{p}$ that have the same numerical value as $l_{p}$ but define two additional different length scales, relating lengths with time units as well as length with mass units. The introduction of basic physical units is in contradiction to classical physics that allows infinite divisibility. As a consequence, measurements in classical physics are impossible, since units cannot be defined. Consequently, Nature could not provide any elemental building blocks to construct higher organized structures, which is inconsistent with observation. Thus the quantiza- 
tion principle is fundamental for the existence of physical objects. Therefore the three Planck length units as defined above must occur in the structure of both spacetime and internal space $\mathrm{H}^{8}$. In spacetime length unit $l_{\mathrm{p}}$ is the basic unit for the spatial coordinates and $l_{\mathrm{pt}}$ measures the time coordinate. In order to connect geometry with physical entities, in the internal symmetry space coordinates $\xi^{i}$ are measured in units of $l_{p m}$. Hence all lengths in $\mathrm{H}^{8}$ are represented by multiples of $1 / m_{\mathrm{p}}$, and therefore internal coordinates $\xi^{i}$ are denoted as energy coordinates. In other words, the concept of energy coordinate ensures that an inverse length is representing a physical mass. Since length values are quantized, the same holds for physical mass. In this regard the connection of geometry with physical objects has been established, but, in order to achieve this goal, the quantization principle had to be introduced $a b$ initio.

In contrast to Einstein, EHT is based on the following four simple and general principles, termed the $G O D Q$ principle of Nature ${ }^{7}$

\section{i. Geometrization principle for all physical inter-} actions,

ii. Optimization (Nature employs an extremum principle),

iii. Dualization (duality, symmetry) principle (Nature dualizes or is asymmetric, bits),

\section{iv. Quantization principle (Nature uses integers only, discrete quantities).}

From the duality principle, the existence of additional internal symmetries in Nature is deduced, and thus a higher dimensional internal symmetry space should exist, which will now be determined.

In $G R$ there exists a four dimensional spacetime, comprising three spatial coordinates, $x^{1}, x^{2}, x^{3}$ with positive signature $(+)$ and the time coordinate $x^{4}$ with negative signature $(-)$. It should be remembered that the Lorentzian metric of $\mathbb{R}^{4}$ (actually spacetime is a manifold $\mathrm{M}^{4}$ ) has three spatial (+ signature) and one time-like coordinate (- signature) [19]. The plus and minus signs refer to the metric that is, the spatial components are taken to be positive and the time coordinate is negative. Therefore, the squared proper time interval is taken to be positive if the separation of two events is less than their spatial distance divided by $\mathrm{c}$. Hence a general coordinate system in $\mathbb{R}^{4}\left(\mathrm{M}^{4}\right)$ comprises the curvilinear coordinates ${ }^{8} \mathrm{x}^{\mu}$ with $\mu=1, . ., 4$. Next, the coordinate structure of $\mathrm{H}^{8}$ is determined. Coordinates in $\mathrm{H}^{8}$ are denoted as $\xi^{i}$, and are termed internal coordinates with $v=1, \ldots, 8$. This set of 8 coordinates will now be

7 This will be discussed in detail in our forthcoming paper: Field propulsion I: Novel Physical Concepts for Space Propulsion.

8 coordinates $x^{\mu}$ can also be Cartesian. Meaning of coordinates will be clear from the context. determined by utilizing the $G O D Q$ principle introduced above. To this end, the second law of thermodynamics is considered, which predicts the increase of entropy. Everywhere in Nature, however, highly organized structures can be found like galaxies, solar systems, planets, plants etc., which, according to the duality principle, have to be introduced into a unified theory. A description of Nature that only provides a route to decay or to lower organizational structures is in contradiction to observation. Therefore, an additional, internal (negative signature-) coordinate, termed entelechial coordinate, $\xi^{5}$, is introduced. The entelechial dimension can be interpreted as a measure of the quality of time varying organizational structure (inverse or dual to entropy). It should be mentioned that all other additional internal coordinates have negative signature, too. Second, when the universe was set into motion, it followed a path marked by a state of great order. Therefore, to reflect this generic behavior in Nature, the aeonic dimension, $\xi^{6}$, is introduced that is interpreted as a steering coordinate toward a dynamically stable state. On the other hand, the entropy principle is firmly established in physics, for instance in

$\beta$ - decay. Entropy is directly connected to probability, which in turn is related to information. Therefore, two additional coordinates $\xi^{7}, \xi^{8}$ are needed, which are complementary to the organizational coordinates, to reflect this behavior of Nature, termed information coordinates that are describing information waves. Finally, since both space and time are essential in the evolution and decay of structures, the internal symmetry space possesses a total of 8 coordinates. In summary, coordinates $\xi^{v}$ with

$v=1, \ldots, 4$ denote spatial and temporal coordinates,

$\xi^{v}$ with $v=5,6$ denote entelechial and aeonic coordinates, and $\xi^{v}$ with $v=7,8$ denote information coordinates in $\mathrm{H}^{8}$. The name gravitophoton has been chosen because of the type of interaction, namely a transformation of the electromagnetic field (photon) into the gravitational field (gravitophoton). The arrow between the gravtitophoton and electromagnetic boxes indicates the interaction between the messenger particles that is, photons can be transformed into gravitophotons. In the same way the quintessence interaction can be generated from gravitons and positive gravitophotons (repulsive force). Heim space, $\mathrm{H}^{8}$ comprises four subspaces, namely $\mathbb{R}^{3}, \mathrm{~T}^{1}$, $\mathrm{S}^{2}$, and $\mathrm{I}^{2}$. In the set of metric-subspaces that can be constructed, where each element is denoted as a hermetry form. Each hermetry form has a direct physical meaning, for details see refs. [9], [20]. In order to construct a hermetry form, either internal space $\mathrm{S}^{2}$ or $\mathrm{I}^{2}$ must be present. In addition, there are three degenerated hermetry forms that describe partial forms of the photon and the quintessence potential. They allow the conversion of photons 
into gravitophotons as well as of gravitophotons and gravitons into quintessence particles. There exist 15 hermetry forms, six of them describe the messenger particles of the fundamental interactions. Hermetry forms $\mathrm{H} 5$, $\mathrm{H} 11$, and H12 are used to describe the gravitational messenger particles. In a very recent announcement by the European Space Agency, 23 March 2006, the measurement of an artificial gravitational field was reported, generated by a rotating superconducting ring.

\section{Propulsion Concepts from Spacetime Physics}

\subsection{Metric Transformation (Transmutation)}

In a recent experiment, funded by the European Space Agency and the Air Force Office of Scientific Research, Tajmar et al. ref. [15] report on the generation of a toroidal (tangential, azimuthal) gravitational field in a rotating accelerated (time dependent angular velocity) superconducting ring. This would be the first time that an artificial gravitational field is generated and, if correct, would have great impact on future technology. Furthermore, the experiment would demonstrate the conversion of electromagnetic interaction into a gravitational field. This is exactly the effect that is predicted by $E H T$, and both a qualitative and quantitative explanation of this effect will be given below. Since the experiment generates a tangential gravitational field, it cannot be used directly as a propulsion system. It is, however, of great importance, since it shows for the first time that a gravitational filed can be generated other than by the accumulation of mass. In this section we will also discuss the validity of the physical explanation, namely the Higgs mechanism to be responsible for the graviton to gain mass, given by Tajmar and de Matos [15], which they termed the gyromagnetic London effect. According to these authors, this effect is the physical cause for the existence of the measured gravitational field.

The arguments of these authors are ingenious, but there is some doubt whether the linearized Einstein equations, see Eqs. $(1,2)$, can be used in the explanation of this effect. Although these equations are predicting such a phenomenon, the effect is 20 orders of magnitude smaller than the observed one, and thus would be completely unobservable. In the derivation, a magnetic field is set equivalent to a gravitational field. This assumption of transforming a magnetic field into a gravitational field is not compatible with current physics.

Instead, the Heim-Lorentz force, as predicted by EHT but now using a coupling to bosons (Cooper pairs), is used to explain this effect. Deriving this effect from gravitophoton interaction, a physical interpretation can be given that explains both qualitatively and quantitatively the experimental results. Moreover, theoretical considerations obtained from EHT lead to the conclusion that a modified experiment will generate a gravitational field acting parallel to the axis of rotation of the ring (torus), see
Fig. 3, and thus can serve as a field propulsion principle $^{9}$. In this experimental configuration the superconducting rotating ring is replaced by an insulating disc and a set of superconducting coils as depicted, in principle, in Fig. 3. EHT allows to calculate the acceleration force and also provides the guidelines for the construction of a propulsion device. The actual experimental setup differs from the simple configuration of the cover picture, but the principle remains unchanged. According to the predictions of EHT experimental requirements, i.e., magnetic field strength, current densities and number of turns of the solenoid, are substantially lower than for fermion coupling that was assumed in all our papers so far, see refs. [9], [10], [20].

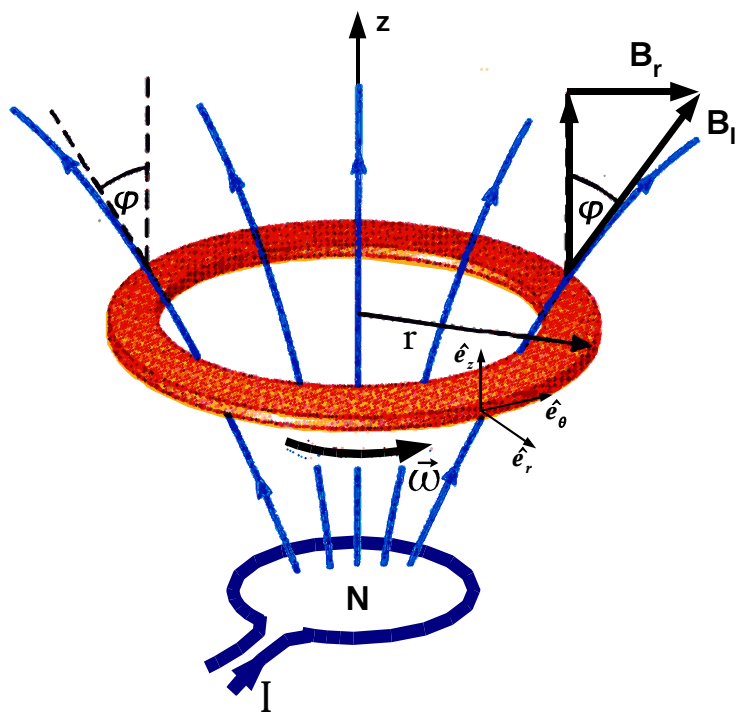

Figure 3: The picture shows the physical principle of the experimental setup to generate a gravitational field in the z-direction (upward, above rotating disk) by the Heim-Lorentz force using a superconducting coil (boson coupling) and a rotating disk or ring. The actual experiment would be somewhat different.

The superconducting current loop (blue), see Fig. 3, provides an inhomogeneous magnetic field at the location of the rotating disk (red). The $z$-component of the gravitophoton field, $b_{z}$ is responsible for the gravitational field above the disk. This experimental setup also serves as the field propulsion device, if appropriately dimensioned.

\subsubsection{Description of the Gravitomagnetic Field Experiment}

The materials for which a strong gravitational acceleration was measured were niobium $\left(\mathrm{Nb}, \mathrm{T}_{\mathrm{C}}=9.4 \mathrm{~K}\right)$ and lead $\left(\mathrm{Pb}, \mathrm{T}_{\mathrm{C}}=7.2 \mathrm{~K}\right)$. No gravitational field was measured in YBCO (Yttrium barium copper oxide, $\mathrm{YBa}_{2} \mathrm{Cu}_{3} \mathrm{O}_{7-\mathrm{x}}, \mathrm{T}_{\mathrm{C}}=$ $94 \mathrm{~K}$ ) and $\mathrm{BSCCO}$ (Bismuth strontium calcium copper oxide, $\mathrm{Bi}_{2} \mathrm{Sr}_{2} \mathrm{Ca}_{n} \mathrm{Cu}_{\mathrm{n}+1} \mathrm{O}_{2 \mathrm{n}+6}, \mathrm{~T}_{\mathrm{C}}=107 \mathrm{~K}$ ) which are so called high-temperature superconductors whose critical

9 A detailed discussion will be given in our forthcoming paper entitled Artificial Gravitational Fields. 
current density is substantially lower than that for $\mathrm{Nb}$ or $\mathrm{Pb}$.

Considering the Einstein-Maxwell formulation of linearized gravity, a remarkable similarity to the mathematical form of the electromagnetic Maxwell equations can be found. In analogy to electromagnetism there exist a gravitational scalar and vector potential, denoted by $\Phi_{g}$ and $\boldsymbol{A}_{g}$, respectively, see [21]. Introducing the gravitoelectric and gravitomagnetic fields

$$
\boldsymbol{e}=-\nabla \Phi_{g} \text { and } \boldsymbol{b}=\nabla \times \boldsymbol{A}_{g}
$$

the gravitational Maxwell equations can be written in the form

$$
\begin{gathered}
\nabla \cdot \boldsymbol{e}=-4 \pi G \rho, \nabla \cdot \boldsymbol{b}=0 \\
\nabla \times \boldsymbol{e}=0, \nabla \times \boldsymbol{b}=-\frac{16 \pi G}{c^{2}} \boldsymbol{j}
\end{gathered}
$$

where $\boldsymbol{j}=\rho \boldsymbol{v}$ is the mass flux and $G$ is the gravitational constant ${ }^{10}$. The field $\boldsymbol{e}$ describes the gravitational field form a stationary mass distribution, whereas $\boldsymbol{b}$ describes an extra gravitational field produced by moving masses.

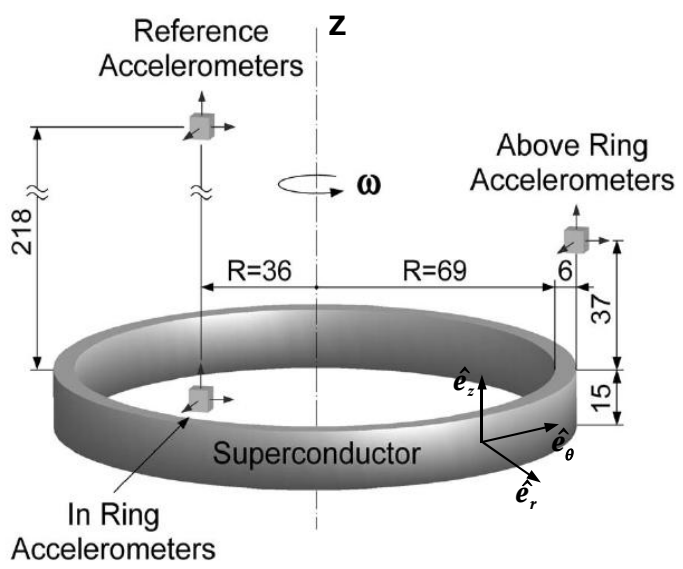

Figure 4: Rotating superconducting torus (Niobium) modified from Tajmar et al. All dimensions are in $\mathrm{mm}$. A cylindrical CS $(r, \theta, z)$ with origin at the center of the ring is used. In Ring accelerometers measured a gravitational acceleration of some $100 \mu \mathrm{g}$ in the azimuthal (tangential, $\theta$ ) direction when the ring was subject to angular acceleration, $\dot{\boldsymbol{\omega}}$. No acceleration was measured in the $z$-direction (upward). If the direction of rotation is reversed, the acceleration field changes sign, too.

At critical temperature $\mathrm{T}_{\mathrm{c}}$ some materials become superconductors that is, their resistance goes to 0 . Superconductors have an energy gap of some $E_{g} \approx 3.5 \mathrm{kT}_{\mathrm{c}}$. This energy gap separates superconducting electrons below from normal electrons above the gap. At temperatures be-

10 Here no consideration is given to the fact that $G$ comprises three parts according to $E H T$. low $\mathrm{T}_{\mathrm{c}}$, electrons are coupled in pairs, called Cooper pairs, which are bosons. The exact formation of Cooper pairs is not known. The coupling of the electron pairs seems to be via phonons, generated by electron movement through the lattice of the superconductor. The size of a Cooper pair is some $10^{3} \mathrm{~nm}$. The crystal lattice contains defects that lead to an energy transfer $\Delta \mathrm{E}$ from the electron gas to the lattice. $\Delta \mathrm{E}$ must be smaller than $\mathrm{E}_{\mathrm{g}}$ otherwise the Cooper pairs are destroyed.

The speed of the Cooper pairs can be calculated in a coordinate system where the electron gas is at rest and the lattice is moving, applying classical energy and momentum conservation. Decelerating the grid means that Cooper pairs gain energy. The maximum amount of energy that a

Cooper pair can absorb is $E_{\text {gap }}$, otherwise it is lifted in the band above, and superconductivity is lost. Therefore the simple ansatz for the maximum energy gap

$1 / 2 m v_{c}^{2}=E_{\text {gap }}=3.5 k T_{c}$ can be used, $v_{c}$ denoting the velocity of a Cooper pair. At temperature $\mathrm{T}_{\mathrm{c}}=10 \mathrm{~K}$ a speed of $v_{c}=10^{4} \mathrm{~m} / \mathrm{s}$ is obtained. A smaller band gap therefore cause a decrease in the speed of the Cooper pairs. Quantum mechanics calculations yield a more correct value of some $v_{c}=10^{5} \mathrm{~m} / \mathrm{s}$.

\subsubsection{Field Propulsion}

Fig. 3 describes the experimental setup for which an insulating disk rotates above a superconducting solenoid. Fig. 4 depicts the experiment of Tajmar et al., where a superconducting ring is subject to angular acceleration. In both cases a gravitophoton force arises. EHT makes the following predictions for the gravitational fields generated by the gravitophoton force.

- In the first case, the gravitophoton force produces a gravitational force above the disk in the zdirection upward and also in the radial direction.

- In the second case, the gravitophoton force is in the azimuthal direction only (experiment by Tajmar et al.).

It is well known that a rotating superconductor generates a magnetic induction field, the so called London moment

$$
\boldsymbol{B}=-\frac{2 m_{e}}{e} \boldsymbol{\omega}
$$

where $\omega$ is the angular velocity of the rotating ring and $e$ denotes elementary charge. It should be noted that the magnetic field in Tajmar's experiment is produced by the rotation of the ring, and not by a current of Cooper pairs that are moving within the ring. This is a major difference between the experiment of Fig. 3 and the proposed experiment depicted in Fig. 4. Therefore the velocity of the Cooper pairs with regard to the lab system is given by $r \omega$ in the experiment of Fig. 3 while the maximal velocity of Cooper pairs in Fig. 4 is given by the maximum energy 
gap, respectively its quantum mechanical counterpart. The major difference between the two experiments lies in the generation of the magnetic induction field $\boldsymbol{B}$. Tajmar and his colleagues simply postulate an equivalence between the generated $\boldsymbol{B}$ field, Eq. (3) with a gravitational field by proposing a so called gravitomagnetic London effect. However, this transformation between electromagnetics and gravitation is introduced ad hoc and contradicts current physics, since the four known physical forces do not allow such a direct coupling.

On the other hand, this kind of coupling is a basic fact of $E H T$, because of its six fundamental interactions, which foresee such a conversion of hermetry form $\mathrm{H}_{7}$, describing photons, into the hermetry form $\mathrm{H}_{5}$, describing the gravitophoton interaction.

Let $R$ denote the radius of the rotating ring, then Eq. 3 puts a limit on the maximal allowable magnetic induction, $B_{\max }$, which is given by

$$
B_{\max }^{2}=14 \frac{m_{e}}{e^{2}} \frac{k_{B} T_{C}}{R^{2}} .
$$

If the magnetic induction exceeds this value, the kinetic energy of the Cooper pairs exceeds the maximum energy gap, and the Cooper pairs are destroyed. The rotating ring is no longer a superconductor. Moreover, the magnetic induction must not exceed the critical value $\boldsymbol{B}_{\mathrm{C}}(T)$, which is the maximal magnetic induction that can be sustained at temperature $T$, and is dependent on the material. EHT predicts that the magnetic induction field $\boldsymbol{B}$ is equivalent to a gravitophoton (gravitational) field $\boldsymbol{b}_{\mathrm{gp}}$. Therefore, the following relation holds, provided that $B$ is smaller than $B_{\max }$

$$
\boldsymbol{b}_{g \boldsymbol{p}} \propto \frac{B}{B_{\max }} \boldsymbol{B}
$$

As soon as $B$ exceeds $B_{\max }$ the gravitophoton field vanishes. From EHT the following general relationship between a magnetic and the neutral gravitophoton field, $\boldsymbol{b}_{g p}$, can be derived

$$
\boldsymbol{b}_{g p}=\left(\frac{1}{(1-k)(1-k a)}-1\right) \frac{e}{m_{e}} \frac{B}{B_{\max }} \boldsymbol{B}
$$

where $k=1 / 24$ and $a=1 / 8$. The dimension of $\boldsymbol{b}_{g p}$ of is $\mathrm{s}^{-1}$. Inserting Eq. 3 into Eq. 6, using Eq. 5, and differentiating with respect to time, results in

$$
\frac{\partial \boldsymbol{b}_{\boldsymbol{g} \boldsymbol{p}}}{\partial t}=\left(\frac{1}{(1-k)(1-k a)}-1\right) \frac{2 e}{m_{e}} \frac{B}{B_{\max }} \frac{\partial \boldsymbol{B}}{\partial t} .
$$

Integrating over an arbitrary area $\boldsymbol{A}$ yields

$$
\int \frac{\partial \boldsymbol{b}_{g p}}{\partial t} \cdot d \boldsymbol{A}=\oint \boldsymbol{g}_{g p} \cdot d \boldsymbol{s}
$$

where it was assumed that the gravitophoton field, since it is a gravitational field, can be separated according to Eqs. $(1,2)$. Since the above formulas will be applied to the experimental configurations depicted in Figs. 3 and 4, cylindrical coordinates $r, \theta, z$ are employed. $\boldsymbol{g}_{\mathrm{gp}}$ is the acceleration field generated by the gravitophoton field. Combining Eqs. 7 and 8 gives the following relationship

$$
\oint \boldsymbol{g}_{g \boldsymbol{p}} \cdot d \boldsymbol{s}=\left(\frac{1}{(1-k)(1-k a)}-1\right) \frac{2 e}{m_{e}} \int \frac{B}{B_{\max }} \frac{\partial \boldsymbol{B}}{\partial t} \cdot d \boldsymbol{A}
$$

From Eq. 3 one obtains

$$
\frac{\partial B}{\partial t}=-\frac{2 m_{e}}{e} \dot{\boldsymbol{\omega}}
$$

Next, we apply Eqs. 9 and 10 to the experimental configuration of Fig. 3, calculating the gravitophoton acceleration for the in-ring accelerometer. It is assumed that the accelerometer is located at distance $r$ from the origin of the coordinate system. From Eq. 3 it can be directly seen that the magnetic induction has a $z$-component only. From Eq. 9 it is obvious that the gravitophoton acceleration is in the $r-\theta$ plane. Because of symmetry reasons the gravitophoton acceleration is independent on the azimuthal angle $\theta$, and thus only has a component in the circumferential (tangential) direction, denoted by $\hat{\boldsymbol{e}_{\theta}}$. Since the gravitophoton acceleration is constant along a circle with radius $r$, integration is over the area $\boldsymbol{A}=\pi r^{2} \hat{\boldsymbol{e}}_{z}$. Inserting Eq. 10 into Eq. 9, and carrying out the integration the following expression for the gravitophoton acceleration is obtained

$$
g_{g p}=-\frac{1}{10} \frac{B}{B_{\max }} \dot{\omega} r
$$

where the minus sign indicates an acceleration opposite to the original one and it was assumed that the $\boldsymbol{B}$ field is homogeneous over the integration area. Now the experimental values taken from the paper by Tajmar et al. will be inserted. The following values are used:

$$
\dot{\omega}=10^{3} \mathrm{rad} / \mathrm{s}^{2}, r=3.6 \times 10^{-2} \mathrm{~m}, B / B_{\max }=3.97 \times 10^{-4}
$$

where the angular acceleration was determined from the slope fit of Fig. 6 in ref. [15] and the $r$ value was determined from Fig. $4(R=36 \mathrm{~mm})$. The ratio of the magnetic fields was calculated from the following formula, obtained by dividing Eq. 3 by the square root of Eq. 4

$$
\frac{B}{B_{\max }}=\frac{1}{\sqrt{7}}\left(\frac{m_{e}}{k_{B} T_{C}}\right)^{1 / 2} \omega R .
$$

Inserting an estimated average value of $\omega=175 \mathrm{rad} / \mathrm{s}$, $m_{e}=9 \times 10^{-31} \mathrm{~kg}, k_{B}=1.38 \times 10^{-23} \mathrm{~J} / \mathrm{K}, T_{C}=9.4 \mathrm{~K}$, and $R=7.2 \times 10^{-2} \mathrm{~m}$, this ratio is calculated as $3.97 \times 10^{-4}$. From Fig. 6 in ref. [15] an experimental value of about $1.0 \times 10^{-4}$ 
$g$ was determined. Inserting the proper values into Eq. 11 finally delivers the theoretical value of the gravitophoton acceleration for the experiment by Tajmar et al.

$$
g_{g p}=1.3 \times 10^{-4} g
$$

Compared to the theoretical value of Eq. 13 there is a close agreement between the predicted gravitophoton force and the measured acceleration. It should be kept in mind that the exact angular velocity was not known and an average value of $175 \mathrm{rad} / \mathrm{s}$ was used. Considering both the mathematical and physical complexity of the derivation the closeness of theory and experiment is remarkable. The results might need to be adjusted for the exact experimental values. It should be noted that values of $k$ and $a$ have been derived some ten years ago and are published in [22]. No parameter was adjusted in the derivation of Eq. 11. Moreover, the theory also correctly predicts direction and sign of the acceleration field. This is seen as a sign that the predicted six fundamental interactions may exist in Nature.

\subsubsection{Space Device Based on Field Propulsion}

The experiment by Tajmar et al. generates an azimuthal gravitational field, and thus is not suitable for propulsion. The lesson learned from the experiment by Tajmar et al. is the fact that the coupling to bosons (Cooper pairs) is of prime importance. However, employing the general Heim-Lorentz force equations to the experimental setup of Fig. 3, Heim-Lorentz force now produces force components in the radial $r$ and $z$-directions. These components are given by

$$
\begin{gathered}
F_{r} \hat{\boldsymbol{e}_{r}}=\frac{v_{C}}{c} m_{e}\left(v_{\theta}^{T} b_{z} \hat{\boldsymbol{e}_{\theta}} \times \hat{\boldsymbol{e}_{z}}\right) \\
F_{z} \hat{\boldsymbol{e}_{z}}=\frac{v_{C}}{c} \frac{v_{\theta}^{T}}{c} m_{n} v_{\theta}^{T} b_{z}\left(\hat{\boldsymbol{e}_{\theta}} \times \hat{\boldsymbol{e}_{z}}\right) \times \hat{\boldsymbol{e}_{\boldsymbol{\theta}}}
\end{gathered}
$$

where $v_{C}$ is the velocity of the Cooper pairs in the superconducting solenoid (Fig. 3), $v_{\theta}^{T}=10 \mathrm{~m} / \mathrm{s}$ denotes the velocity of the rotating disk or ring, and $b_{z}$ is the component of the (gravitational) gravitophoton field $\boldsymbol{b}_{g p}$ (dimension $1 / s$ ) in the z-direction. In contrast to the fermion coupling, experimental requirements are modest. The following assumptions were made: $N=100$ number of turns of the solenoid, current of some 1-2 $A$ (needed to calculate $b_{\mathrm{z}}$ ), diameter of solenoid $0.1 \mathrm{~m}$. A detailed analysis predicts an acceleration in $z$-direction of some $6.0 \times 10^{-5} \mathrm{~g}$. From these numbers it is clear that, if theoretical predictions are correct, the realization of a workable space propulsion device that can lift itself from the surface of the Earth seems to be feasible.

\section{Conclusions and Future Work}

In this paper an overview of the current status of space propulsion was given. It has been shown that even with an advanced fission propulsion system, space travel will be both very limited and very costly. Travel time to other planets will remain high. Metric engineering of spacetime or using wormholes does not seem to be technically feasible. On the other hand, the recent experiment by Tajmar, if confirmed, has shown that a coupling between electromagnetism and gravitation exists, which allows the generation of artificial gravitational fields. EHT, which uses an internal 8-dimensional space, has predicted this kind of coupling and foresees six fundamental physical interactions. The theory was successfully applied to predict the outcome of Tajmar's experiment and also provides guidelines for an experimental setup that can be used as field propulsion device. Research therefore should focus on the refinement of the experiment as well as on the theoretical foundation of EHT.

\section{References}

1. Villard, R., L.R. Cook: Infinite Worlds, Univ. California Press, , 2005.

2. Zaehringer, A.: Rocket Science, Apogee Books, Chap 7., 2004.

3. Mallove, E. and G. Matloff: The Starflight Handbook, Wiley, Chap. 3, 1989.

4. Jahnshan, S.N. and T. Kammash: Multimegawatt Nuclear Reactor Design for Plasma Propulsion Systems, Vol 21, Number 3, May-June 2005, pp.385-391.

5. Emrich, W.J. and C. W. Hawk: Magnetohydrodynamic Instabilities in a Simple Gasdynamic Mirror Propulsion System, Vol 21, Number 3, May-June 2005, pp.401-407.

6. Czysz, P.A., C. Bruno: Future Spacecraft Propulsion Systems, Springer, 2006.

7. Krauss, L.M.: Propellantless Propulsion: The Most Inefficient Way to Fly?, NASA TM/CP 208694, January 1999.

8. Rovelli, C.: Loop Quantum Gravity, IoPNovember 2003.

9. Dröscher, W., J. Hauser: Heim Quantum Theory for Space Propulsion Physics, pp. 1430-1441, AIP, 2005.

10. Liddle, A.: An Introduction to Modern Cosmology, Wiley, 2003.

11. Witten, E.: Reflection on the Fate of Spacetime, Physics Today, 1996.

12. Hartle, J.B.: Gravity, Addison Wesley, 2003.

13. Vass, R.: Tunnel durch Raum und Zeit, Kosmos, Stuttgart, 2005.

14. Heim, B.: Vorschlag eines Weges einer einheitlichen Beschreibung der Elementarteilchen, Z. für Naturforschung, 32a, pp. 233-243, 1977.

15. Tajmar, M. et al.: Experimental Detection of the Gravitomagnetic London Moment, arXiv, gr-qc/06030332006.

16. Zwiebach, R.: Introduction to String Theory, Cambridge Univ. Press, 2004.

17. Dröscher, W., J. Hauser: Magnet Experiment to Measuring Space Propulsion Heim-Lorentz Force, 41 ${ }^{\text {st }}$ JCP, AIAA 2005- 4321, 10 pp., Tucson, AZ, 10-13 July, 2005.

18. Schiller, C.: Motion Mountain, The Adventure of Physics (Chap. XI), September 2005, www.motionmountain.net.

19. Carrol, S. M.: Spacetime and Geometry, Addison-Wesley, San Francisco, 2004. 
20. Dröscher,W., J. Hauser: Guidelines for a Space Propulsion Device Based on Heim's Quantum Theory, 40 th JCP, AIAA 2004-3700, 21 pp., Ft. Lauderdale, FL, 7-10 July, 2004.

21. Hobson,M.P., Efstathiou, G., and A.N. Lasenby: General Relativity, Cambridge University Press, 2006.

22. Heim, B., Dröscher, W: Strukturen der Physikalischen Welt und ihrer nichtmateriellen Seite, Resch Verlag1996. 